\title{
Design of Band-Pass Filter using Artificial Neural Network
}

\author{
Shushank Dogra \\ M.Tech. Scholar \\ Amritsar College Of Engg. \& Tech \\ Amritsar
}

\author{
Narinder Sharma \\ Associate Professor \\ Amritsar College Of Engg. \& Tech \\ Amritsar
}

\begin{abstract}
For the design of Band pass FIR filters complex calculations are involved. Mathematically, by substituting the value of pass-band ripple, stop band attenuation, pass-band frequency F1, pass-band frequency F2, sampling frequency in any of the methods from window method, frequency sampling method or optimal method we can get the values of filter coefficients $\mathrm{h}(\mathrm{n})$. Here, window method is used in which Kaiser window method has been chosen preferably because of the presence of ripple factor $(\beta)$.Here, I have design Band pass FIR filter using artificial neural network which gives optimum result i.e. the difference between the actual and desired output is minimum.
\end{abstract}

\section{Keywords}

Window functions, Artificial neural network.

\section{INTRODUCTION}

With the technological evolution, great encourage have been built on design techniques for various digital filters. A filter is essentially a system or network that selectively changes the wave shape amplitude - frequency and or phase - frequency characteristics of a signal in a desired manner (Zhao and Yu., 1997). A digital filter is a mathematical algorithm implemented in hardware and/or software that operates on a digital input signal to produce a digital output signal for the purpose of achieving a filtering objective. (Wade et al., 1990) described filters are synthesized by cascading elements from a library of computationally simple and some case very computationally efficient, primitive filter.A simplified block diagram of real time digital filter,with analog input and output signals is shown in Fig 1

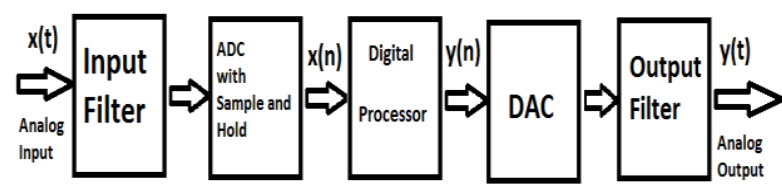

Fig 1: Diagram of digital filter

\subsection{Some of the advantages of digital} filters [Ifeachor] are listed as below:

$\square$ The performance of digital filters does not vary with environmental alters, for example thermal variations.

The frequency response of digital filters can be automatically adjusted if it is implemented using programmable processor.
Several input signals or channels can be filtered by one digital filter without the need to replicate the hardware.

Both filtered and unfiltered data can be maintained for further use

$\square$ The performance of digital filters is repeatable from unit to unit.

$\square$ Digital filters can be used at very low frequencies

The following are the primary disadvantages of digital filters [Ifeachor] compared with analog filters:

Speed Limitation: The maximum bandwidth of signals that digital filters can handle in real time, is a lot let down than for analog filters.

$\square$ Finite word length effects: Digital filters are subject to ADC noise resulting from quantizing a continuous signal, and to round off noise incurred during computation. With higher order recursive filters the accumulation of rounded off noise could lead to instability.

$\square$ Long design and development times: The design and development times for digital filters, especially hardware growth, can be a lot longer than for analog filters.

\subsection{Advantages of FIR filters over IIR}

\section{filters}

1. FIR filters have an exactly linear phase responses where the phase responses of IIR filters are non linear.

2. FIR are realized nonrecursively, that is by direct evaluation are ever stable. The stability of IIR filters cannot ever be guaranteed.

3. The coefficient quantization error is much less severe in FIR than in IIR.

\section{VARIOUS WINDOW FUNCTIONS}

There are many windows proposed that approximate the desired characteristics. The basic window functions are listed below:

Rectangular Window

$\mathrm{W}_{\mathrm{r}}(\mathrm{n})= \begin{cases}1, & \text { for- }(\mathrm{M}-1) / 2 \leq \mathrm{n} \leq(\mathrm{M}-1) / 2 \\ 0, & \text { otherwise }\end{cases}$ 
Bartlett Window

$$
\mathrm{W}_{\mathrm{T}}(\mathrm{n})= \begin{cases}2 \mathrm{n} /(\mathrm{M}-1) & \text { for } 0 \leq \mathrm{n} \leq(\mathrm{M}-1) / 2 \\ 2-2 \mathrm{n} /(\mathrm{M}-1) & \text { for }(\mathrm{M}-1) / 2<\mathrm{n} \leq(\mathrm{M}-1) \\ 0, & \text { otherwise }\end{cases}
$$

This window is also called Barlett window.

\subsection{KAISER WINDOW}

It can be seen that the width of the main lobe [4]is inversely proportional to the length of the filter. As the length of the filter is increased, the width of the main lobe becomes narrower and narrower, and the transition band is cut down substantially. The attenuation of the side lobes is, however, independent of the length and is a function of the type of the window. Hence, a proper window function is to be selected in order to achieve a desired stopband attenuation. A window function with minimum stop band attenuation has the maximum main-lobe width. Hence, the length of the filter must be increased considerably to reduce the main-lobe width and to achieve the desired transition band.The Kaiser window is defined as

$$
W_{k}(n)=\left\{\begin{array}{lr}
I_{0}\left(\beta\left(1-[(n-a) / a]^{2}\right)^{1 / 2}\right) & \text { for } 0 \leq n \leq M-1 \\
0, & \text { otherwise }
\end{array}\right.
$$

Where $\alpha=(\mathrm{M}-1) / 2$ and $\mathrm{I}_{0}$ represents the zeroth-order modified Bessel function of the first kind. The Kaiser window has two parameters... the length (M) and a figure parameter $\beta$, the window length and figure can be adjusted to trade side lobe amplitude for main lobe width. If the window is tapered more, the side lobes of the Fourier transform become smaller, but the main lobe becomes wider. Increasing length $M$ while holding $\beta$ constant causes the main lobe to decrease in width but does not affected the amplitude of the sidelobe.

Kaiser obtained a pair of formulas that permit the filter designers to predict in advance the values of $M$ and $\beta$ needed to meet a given frequency selective filter specifications. Kaiser found that over a usefully wide range of conditions, the peak approximation error $(\delta)$ is determined by the choice of $\beta$. Given that $\delta$ is fixed, the passband cut-off frequency PBF of the low pass filter is defined to be the highest frequency such that $\left|H\left(e^{j \omega}\right)\right| \geq 1-\delta$. The stop band cut-off frequency $\omega_{\mathrm{s}}$ is defined to be the lowest frequency such that $\left|H\left(e^{j \omega}\right)\right| \leq \delta$. Therefore, the transition region has the normalised width

$\mathrm{df}=\mathrm{f}_{\mathrm{s}}-\mathrm{f}_{\mathrm{p}} / \mathrm{f}=\phi_{\mathrm{s}}-\omega_{\mathrm{p}}\left|\left(\begin{array}{l}2 \pi \\ \end{array}\right)\right|$

For the low pass filter approximation. Defining

$\delta=\operatorname{minimum}\left(\delta_{\mathrm{p}}, \delta_{\mathrm{s}}\right)$

Attenuation $\mathrm{A}=20 \log _{10} \delta \mathrm{dB}$

Kaiser determined empirically that the value of $\beta$ needed to achieve a specified value of $A$ is given by

$$
\beta= \begin{cases}0.1102(A-8.7) & \text { for } A>50 \\ 0.5842(A-21)^{0.4}+0.07886(A-21) & \text { for } 21 \leq A \leq 50 \\ 0.0 & \text { for } A<21\end{cases}
$$

Further more, to achieve prescribed values of $\mathrm{A}$ and $\mathrm{df}, \mathrm{M}$ must satisfy

$$
\begin{aligned}
& \mathrm{M}=\frac{A-8}{14.36 d f}+1 \quad \text { for } \mathrm{A}>21 \\
& (0.922 / d f)+1 \quad \text { for } \mathrm{A} \leq 21
\end{aligned}
$$

Finite Impulse response filters (One and paper.,1999) are preferred for their stable and linear phase feature. But due to pant impulse response of FIR filters there will be more hardware complexity.

\section{ARTIFICIAL NEURAL NETWORK}

An Artificial Neural Network is an information processing paradigm inspired by the way the densely inter-connected, parallel structure of the mammalian brain process information. ANN have successfully applied to a number of problems including the identification and control of dynamical systems, communications networks, coordination of robotics hand eye movements. It is also referred to as a neuromorphic system, follows connectionist architecture, and parallel distributed processing. Artificial Neural Networks are collections of mathematical models that emulate some of the observed properties of biological nervous system. The key element of the ANN is the novel structure of the information processing systems.

Some other advanrtages of ANN are as under:

1. Adaptive learning

2. Self-Organisation

3. Real Time Operation

\subsection{Multilayer Perceptron Networks[3],[4]}

Multilayer Perceptron Networks form a class of feed forward neural networks. They are not a single layer network but consist of an input layer, arbitrary number of hidden layers and an output layer as shown in figure 1.5.Here input is fed to each of the input layer neurons. The outputs of the input layer feed into each of next layer neurons and so on, forming a layered structure having one input layer, one output layer and L-2 hidden layers in an L layer network. The following diagram illustrates a perceptron network with three Layers: 


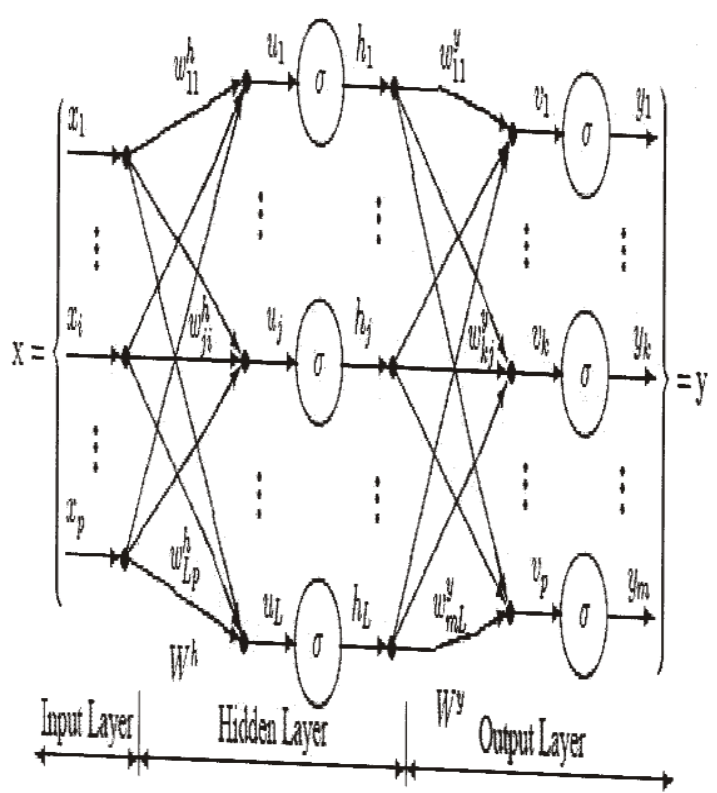

Fig.2: Multilayer Perceptron

\subsection{Formulation of Problem}

The design of digital filter means basically finding the values of filter coefficients so that given filter specification are achieved the window based design method are exclusively used for calculating there coefficient. We have used Kaiser window for this purpose. The Kaiser window function goes somehow in overcoming the incorporating a ripple control parameter, ANNs have been used for the design of digital filter with Passband ripple, stop band attenuation, passband frequency F1, passband frequency F2, sampling frequency as input parameters. In this thesis ANN have been used to design the band pass FIR filter coefficient that are matching with coefficient given by Kaiser with here the multi layer perception feed forward network has been used for the design because this method is efficient, accurate,less complex and easily implemented. The network has been trained in such a manner so that the error comes minimum, means there may be very less difference in the results comes from actual calculations that has been come from Matlab and the output comes from trained artificial neural network.

\subsection{GOAL}

The goal of the present work are divided into the following sections.

(1) To prepare the data sheet using different values of filter parameter achieve the filter coefficient.

Choosing ANN a Band-pass FIR filter has been designed such that its coefficient match with coefficients obtained with window method.

\section{PROBLEM METHODOLOGY}

The major steps are followed in the execution ofthis work.

Step 1: Development of codes in Mathab for the following.

- Ann.m - For data preparation.

- Ann2.m - For training of the neural network

Step 2: By using ann.m code the following are database file in $\mathrm{xls}$ format has been prepared.

- db new_input.xls - Input data information in matrix form

- $\mathrm{db}$ new target.xls -Target information in matrix form

Step 3: Using ann2.m code the training of neural network has been done.

- Preparation of data sheet:- With following that are

- Passband Ripple (Ap)

- Stopband Attenuation (As)

- Pass Band Frequency (F1)

- Pass Band Frequency (F2)

- Sampling Frequency (Fs)

Filter coefficient are calculated and in this thesis works is carried out using approximately 30 such values of all the above parameters to calculate the filter coefficients. The range of different parameters has been taken which are:

- Ap: $(0.7-1.3) \mathrm{db}$

- As: (40-55) db

- F1: (7000-11000) hz

- F2: (17000-21000) hz

- Fs:(47000-52000) hz

Using this data set the Artificial Neural Network has been trained and can be use to calculate filter coefficients for input parameters in this range.Now, ANN is use to design the band pass FIR filter.There is very less difference in the ann results and the calculated results.

\section{RESULT}

The network has been trained using Multilayer Perceptron in which Error Back Propagation Algorithm has been used to design BAND PASS FIR filter.using "Levenberg Marquardt" (trainlm) in the neural network feedforward (newff) the goal meet condition has been achieved as shown in fig. 3 


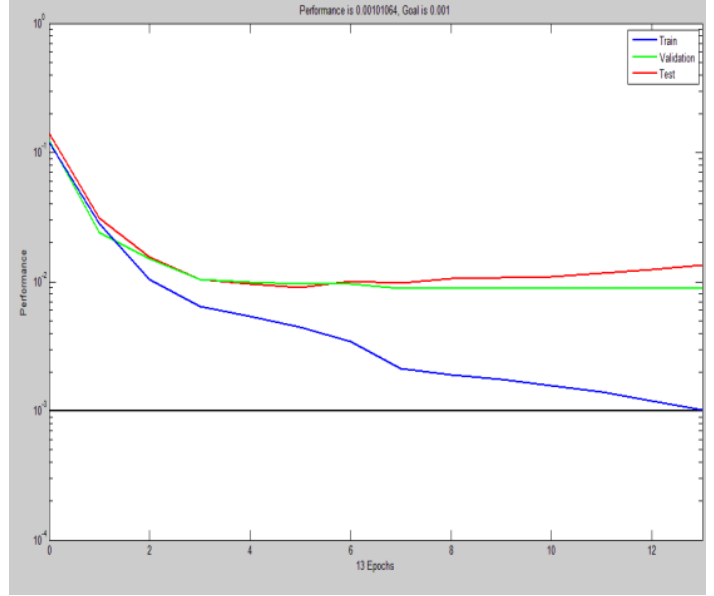

Fig.3:Training of Artificial Neural Network

\subsection{Kaiser vs Artificial Neural Network}

Input parameters:- $A p=0.729 . A s=41.502 \quad F 1=9815$, F2=18234, Sampling frequency $=49661$,Filter Length $=17$

\begin{tabular}{|l|l|l|l|}
\hline $\mathbf{h}(\mathbf{n})$ & $\begin{array}{l}\text { Kaiser } \\
\text { Window } \\
\text { Method }\end{array}$ & $\begin{array}{l}\text { Artificial } \\
\text { Neural } \\
\text { Network }\end{array}$ & $\begin{array}{l}\text { Error } \\
\text { Values }\end{array}$ \\
\hline $\mathrm{h}(0)$ & 0.0001 & -0.2214 & 0.0015 \\
\hline $\mathrm{h}(1)$ & 0.0047 & 0.0058 & -0.0011 \\
\hline $\mathrm{h}(2)$ & 0.0109 & 0.0100 & 0.0009 \\
\hline $\mathrm{h}(3)$ & -0.0204 & -0.0209 & 0.0005 \\
\hline $\mathrm{h}(4)$ & -0.0108 & -0.0101 & -0.0007 \\
\hline $\mathrm{h}(5)$ & 0.0329 & 0.0331 & -0.0002 \\
\hline $\mathrm{h}(6)$ & -0.0019 & -0.0015 & -0.0004 \\
\hline $\mathrm{h}(7)$ & -0.0306 & -0.0306 & -0.0000 \\
\hline $\mathrm{h}(8)$ & 0.0076 & 0.0075 & 0.0001 \\
\hline $\mathrm{h}(9)$ & 0.0001 & 0.0003 & -0.0002 \\
\hline $\mathrm{h}(10)$ & 0.0188 & 0.0185 & 0.0003 \\
\hline $\mathrm{h}(11)$ & 0.0286 & 0.0284 & 0.0002 \\
\hline $\mathrm{h}(12)$ & -0.0906 & -0.0895 & -0.0011 \\
\hline $\mathrm{h}(13)$ & -0.0166 & -0.0175 & 0.0009 \\
\hline $\mathrm{h}(14)$ & 0.1686 & 0.1695 & -0.0009 \\
\hline $\mathrm{h}(15)$ & -0.0560 & -0.0547 & -0.0013 \\
\hline $\mathrm{h}(16)$ & -0.1980 & -0.1982 & 0.0002 \\
\hline $\mathrm{h}(17)$ & 0.1482 & 0.1483 & -0.0001 \\
\hline & & & \\
\hline
\end{tabular}

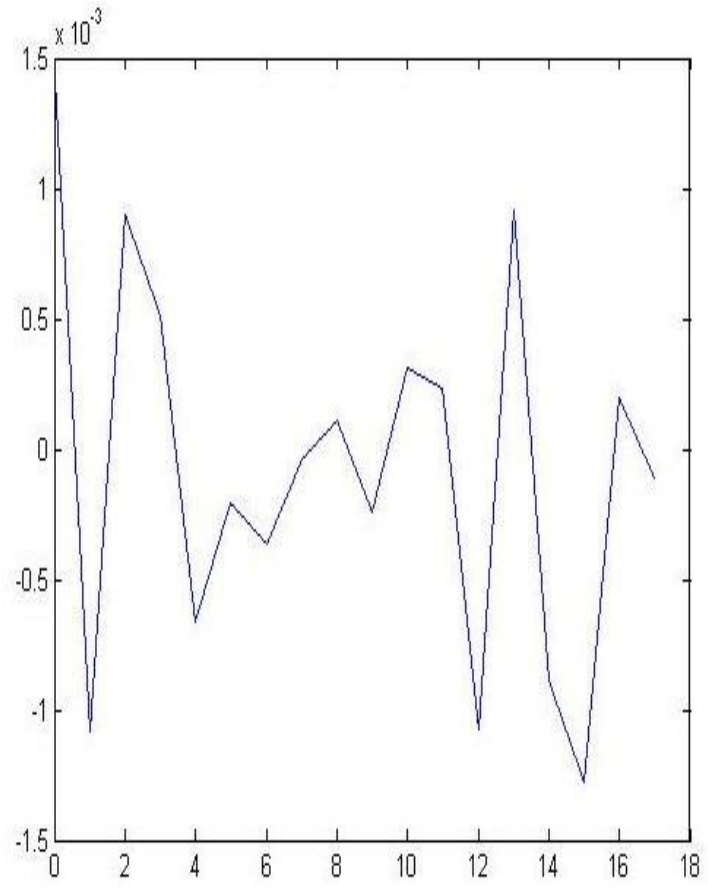

Fig.4:Error graph

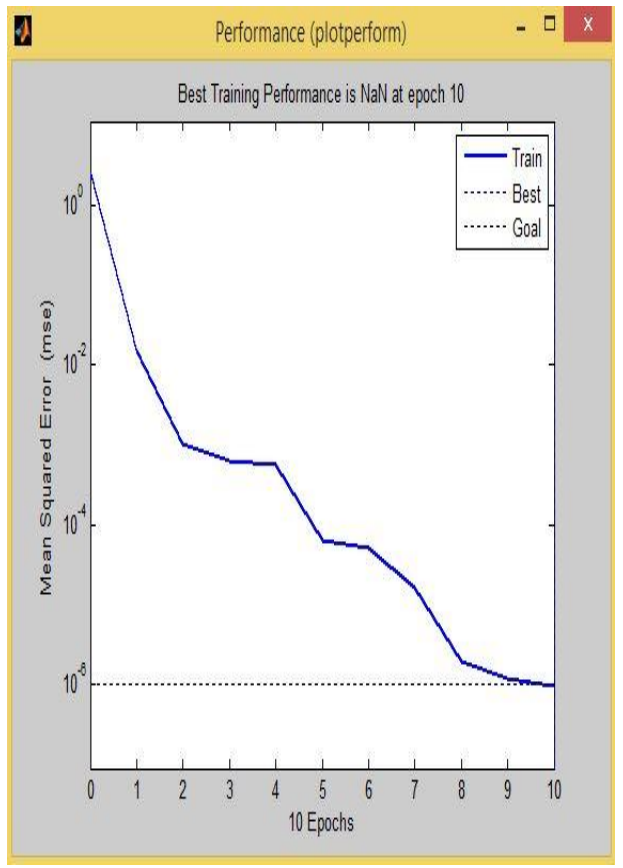

Fig.5:Performance Plot 


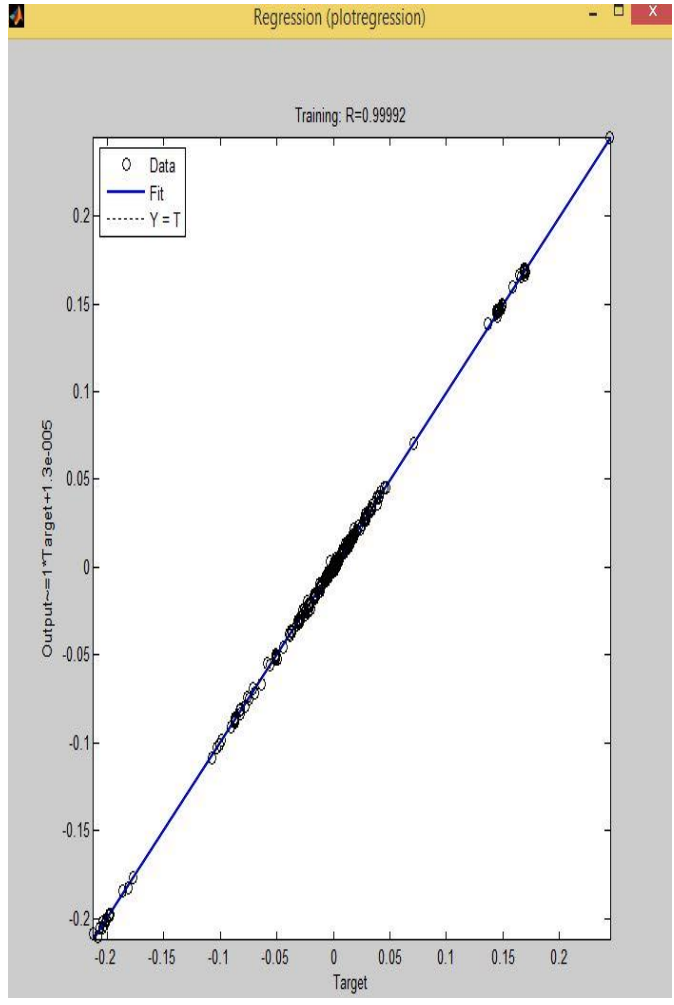

Fig.6:Regression plot

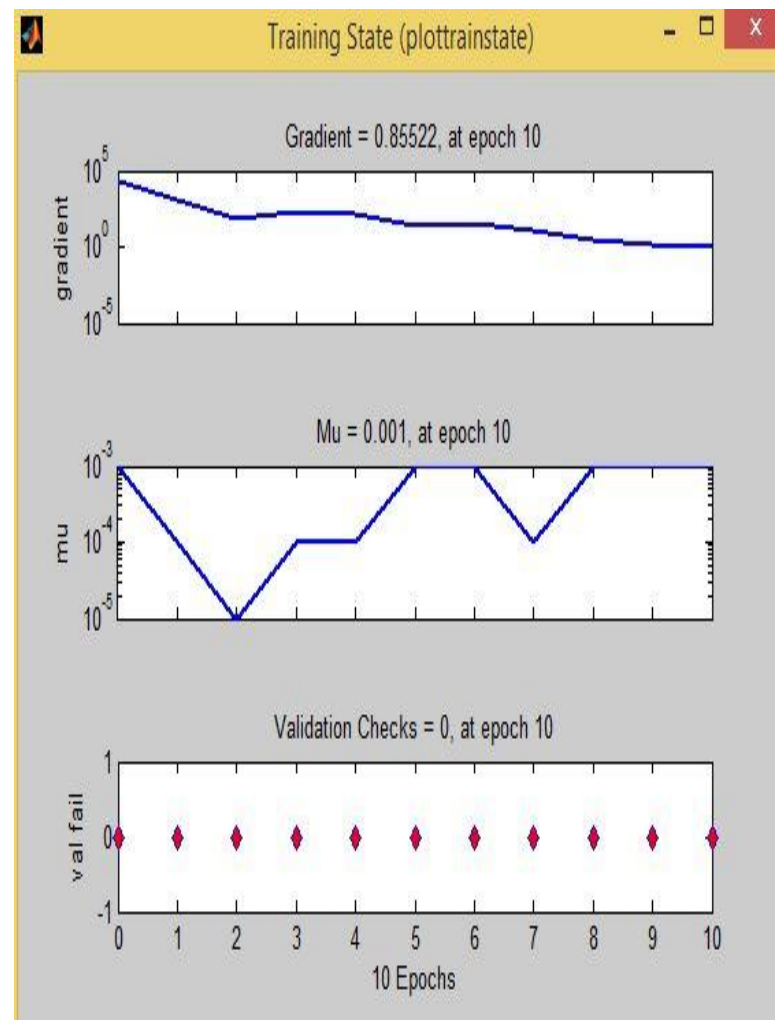

Fig.7:Training states

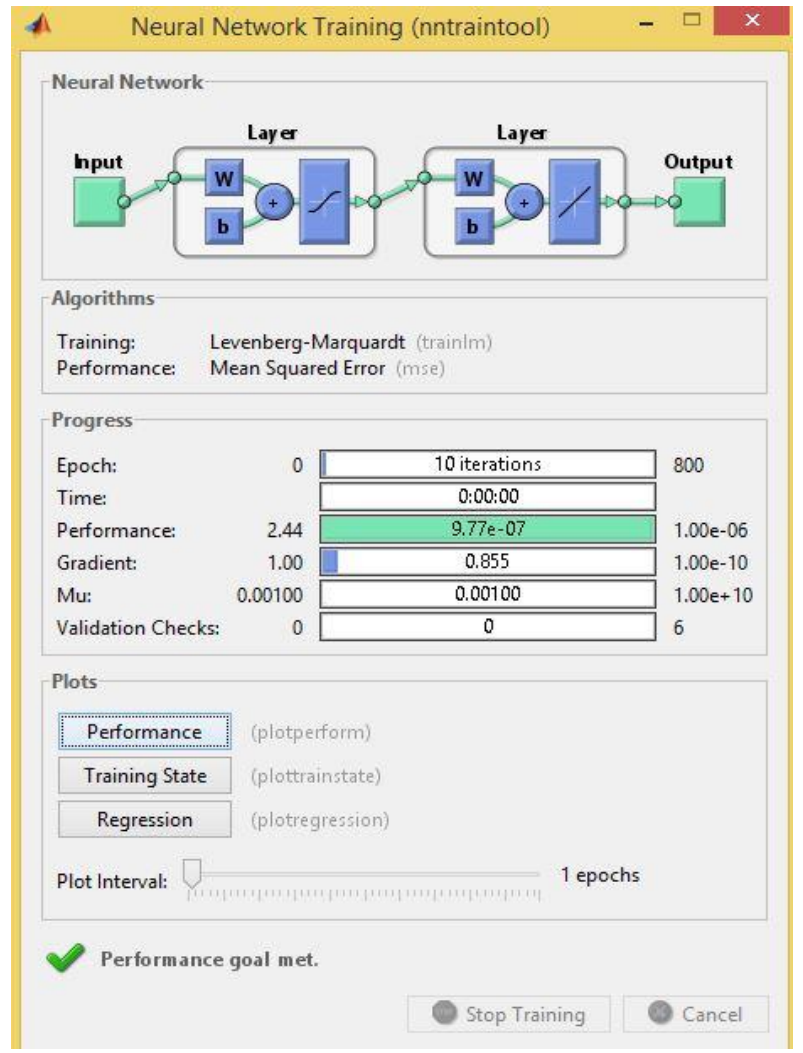

Fig.8:Neural Network Training

\section{CONCLUSION}

The present thesis has illustrated the need of Artificial Neural Network for the design of Band pass FIR Filters rather than using the complex calculative Window method..

The adopting conclusions are drawn from this research work:

- Artificial Neural Network is better and easy method of Design of band Pass FIR Filter.

- Using Fourier series, Frequency sampling or Window methods the filter can be design but for each unknown parameter the filter coefficients have to calculated. In comparison with ANN, the trained network can calculate the filter Coefficient for unknown parameter in that specified range.

- The error graphs shows that results come from Kaiser window method and artificial neural network is very less.

\section{REFERENCES}

[1] V. Aggarwal, J. O. Wesley and M. O Una., (2006) "Filter approximation using Explicit Time and Frequency Remain specification". Procedure, of the Annual Symposium on Artificial Intelligence, 2006, Seattle, Washington, pp. 174-165.

[2] A. M. Cristian and H. Guinther, (2007) "Architecture Optimization of a Finite Impulse Response Filter using togglebased power estimation", International conference, on intelligent and Advance systems, 2007, pp. 12701273. 
[3] A. Fizelow., P. Brites., A. Ochoa., H. Mertuns., E. Fernandez, and Garcia-Martinez R. (2007) "Finding Optimal Neural Network Architecture using Genetic Algorithms," Procedure of the encourages in computer and Engineering, 2007, pp. 15-24.

[4] D. Bhattacharya and A. Antoniou, (1996) "Real Time Design of FIR Filters of Feedback Neural Network", Vol. 3, 1996, pp. 1070-1078

[5] D. A. Yasur and R. Teresa., (2007) "LUT-based Power Macro modeling Technique for DSP architectures", Procedure of the IEEE, Centro de Electronics Industrial, Spain, August, 2007, pp. 1416-1419.

[6] K.B. Englihart, B.S. Hudgins, M. Stevenson and P.A. Parker, (1994) "Myoelectric Signal Classification using a Finite Impulse Response Neural Network". Technical Report, The university of New Brunswork, Canada, June, 1994, pp. 803-820.

[7] Z. Fan and P. Mars, (1997) "Access flow control scheme for ATM networks using neural-network-based traffic prediction". Procedure for IEEE, Vol. 144, Dec. 5, October 1997, pp. 708- 714.

[8] G. Rama Marthy. (2008) "Finite Impulse Response FIR filter Model of Synapses: Associated Neural Network", Procedure of the Fourth Annual IEEE International Confidences on Natural Computation, 2008, pp. 33043309.
[9] G. Zichao and U. E. Robert., (1992) "Using Genetic Algorithms to select input for Neural Network". Procedure of the IEEE International conference, May, 1992, pp. 87-95.

[10] K.J. Hintz. and J.J.Spofford , (1990) "Evoling Neural Network" Procedure of the IEEE Transactions on Communication and Intelligence, May 1990, pp. 333338.

[11] S. Haykins, (2003), "Neural Networks - A comprehensive foundation", Prentice - Hall of India Private Limited, New-Delhi 2003.

[12] I. Ioan., R. Corina and I. Arpad., (2004) "The optimization of feed forward", procedure of the international and data - ICTAMI 2004, Thessalo-niki, Greece.

[13] I F. Emmonual C. and J. Barriel W. (2001) "Digital Signal Processing," A Practical Approch," Person Education (Singapore) Ltd., 2001, Second Edition.

[14] J. Y. Dar. and C. F. Kun., (2007) "Least square Design of FIR Filters based on a compacted Feedback inert Network", Proceeding of the IEEE Transaction on Circuits and systems, vol. 54 issue 5, May, 2007, pp. 427-431. 